\title{
Physical education of children with autism spectrum disorders: a systematic review of structure and effects of interventional programs
}

\author{
DOI: https://doi.org/10.5114/pq.2020.96232
}

\author{
Ivanna Bodnar', Iuliia Pavlova', Ali Khamade ${ }^{1,2}$ \\ ${ }^{1}$ Lviv State University of Physical Culture, Lviv, Ukraine \\ 2 Shafallah Center, Doha, Qatar
}

\section{Abstract}

Introduction. Studies of the effect of physical activity on indicators of physical and social health, as well as communication skills remain relevant owing to the lack of clear recommendations regarding the types, organization, and substantive content of sports and exercise interventional programs, as well as means of physical education (PE) effective for autism spectrum disorders (ASD) treatment and correction and improving the participants' quality of life.

Methods. The electronic databases of PubMed, LILACS, MEDLINE, Embase, and Google Scholar were searched. Co-authors analysed the articles in accordance with a checklist, developed their summary, and verified their accuracy. All the search results are relevant as of June 2019.

Results. In the scientific studies of 2000-2019 concerning the effect of applying PE programs for children with ASD, the participants were usually heterogeneous in age, level of functional capabilities, and intelligence quotient. The duration of the experiment was short (8-14 weeks); the frequency of interventions was different, the training sessions lasted 45-60 minutes. PE activities with proven effectiveness were swimming and water games, running, walking, imitation of riding, elements of yoga and oriental martial arts.

Conclusions. It is worth conducting a randomized study with a representative sample, within a group of the same age, intelligence quotient, daily physical activity, functional capabilities that would investigate indicators of all groups (physical, cognitive, behavioural, communicative) in an integrated manner, with a particular focus on those that are of interest to practice.

Key words: autism spectrum disorders, children, physical activity, program, quality of life

\section{Introduction}

The number of children with autism spectrum disorders (ASD) increases each year [1, 2]. Recent population-based studies have found a high prevalence rate of ASD. It has also been reported that an average of $1 \%$ of children has ASD in the USA; in South Korean schools, the index equals $3.7 \%$ among males and $1.5 \%$ among females [2-4]. It is also emphasized that nearly $2 / 3$ of medical cases remain undiagnosed and therefore do not receive any specialized help or services.

The term 'ASD' stands for a wide range of neurodevelopmental disorders that involve autism disorder, Asperger syndrome, Rett's disorder, childhood disintegrative disorder, and pervasive developmental disorder - not otherwise specified (PDD-NOS). The leading diagnostics features of ASD include limited communication skills, difficulties with social interaction, restrictive behaviour patterns, difficulties in the development of motor behaviour; the diagnosis of specific subtypes requires an overall understanding of cognitive abilities, speech, communication, as well as behavioural, social, and adaptive problems. Behavioural problems may consist in hyperactivity/inattention, aggression, obsessive-compulsive-like behaviours, and sleep disorders [2, 5]. Also, limited motor coordination, deficiencies in fine and gross motor functioning, repetitive, stereotypic movement in extraordinary/ stressful situations are common.

Children with ASD tend to have an inadequate level of physical activity (PA) $[6,7]$; they often present difficulties with balance, posture stability, joint flexibility, and speed qualities development. Usually, this results in the occurrence of diseases, disharmonious physical development, and a low level of physical fitness [8-11]. Among the postponed effects of the reduced PA opportunities are a dangerously sedentary lifestyle, cardiovascular disease, obesity, insulin resistance syndrome, etc. People with ASD constitute a particular risk group, and PA may be a useful tool to prevent some of their health problems.

A special and well-designed system of physical education (PE) can be an effective way to reduce such risks and, therefore, to increase the level of physical fitness, as well as the child's psychomotor, cognitive, and emotional development. Nowadays, it is generally known that PE training and sports have a positive effect on indicators of mental and physical development, as well as children's physical fitness. The increasing number of physical exercises improves physical health, intellectual abilities, behavioural and social skills. Metaanalyses and studies have provided evidence that exercises determine improvement in muscular and cardiovascular endurance; they increase physical strength and flexibility of individuals with intellectual disability. However, much less is known on whether such correlations can be found in children with ASD and to what extent.

There are several meta-studies that confirm the positive effects of various means of PE on the performance of children with ASD, in particular, their cognitive development $[12,13]$, health indicators, behavioural problems (notably, indicators of aggressiveness and destructive behaviour) [13],

Correspondence address: Iuliia Pavlova, Department of Theory and Methods of Physical Culture, Lviv State University of Physical Culture, 11, Kostiushko Str., Lviv, Ukraine, 79007, e-mail: pavlova.j.o@gmail.com 
motor skills, social skills [14], muscle strength and endurance [14], and disruptive behaviour (e.g. stereotypy and aggressive behaviour) [5].

Nowadays, there are several approaches to organizing PE for children with ASD. Some authors [15] are convinced that individual tasks are more significant in improving the psychophysical development and elaboration of social skills among children with ASD. However, in other studies [13], considerable improvements in the studied parameters were revealed during group training.

Research has shown that improved health, including some psychosocial indicators in children with ASD is a result of applying specific types of PA in the developed PE programs [12-15]. Running and swimming were the most common means of PE for this category of children [15]. At the same time, intensive PA brought about stronger effects [13] than training programs with a low level of PA. In general, the importance of PA for individuals with ASD has been confirmed in scientific papers, and the magnitude of the positive effects varies from medium to above average. There is evidence that in children with ASD, PE improves maladaptive behaviour patterns, communication skills, and academic engagement, although not all cognitive processes become enhanced [15].

ASD is a life-long condition; therefore, additional studies on the effect of PA on physical and social health indicators and communication skills remain relevant owing to the lack of clear recommendations regarding the types, organization, and content of sports and exercise interventional programs, as well as means of PE effective in ASD treatment and correction and in improving the program participants' quality of life.

The purpose of this review was to analyse PE programs aimed at correcting the problems of children with ASD, as well as to clarify their informative saturation and logic of construction. The review tasks were: (1) to describe the characteristics (participants, sample size, the availability of control group, etc.) and exercise interventions (type, duration of the program and individual sessions, etc.) of the included studies; (2) to analyse the purposes and effects of the interventional programs, identify their weak points, and determine the general features of programs with proven effectiveness.

\section{Subjects and methods}

The search covered the electronic databases of PubMed, LILACS, MEDLINE, Embase, and Google Scholar. The following key words were used: 'Asperger,' 'autism,' 'ASD/ autistic spectrum disorders,' 'pervasive developmental disorders/PDD-NOS'. These terms were paired with 'physical education,' 'physical culture,' 'physical exercise,' 'physical activity,' 'group exercise'. Selective Boolean operators 'AND' and 'OR' were applied. All the search results are relevant as of June 2019.

\section{Study selection and data extraction}

A PRISMA diagram of the study selection process is provided in Figure 1. To be included into the analysis, the studies had to meet the following criteria: (1) reference solely to children with an ASD diagnosis; (2) the intervention description involving physical exercise, detailed content of the interventional programs, their ways of implementation, and effectiveness; (3) publication in the years of 2000-2019; (4) the full text of the article available in English. Exclusion criteria were as follows: (1) protocols with incomplete studies

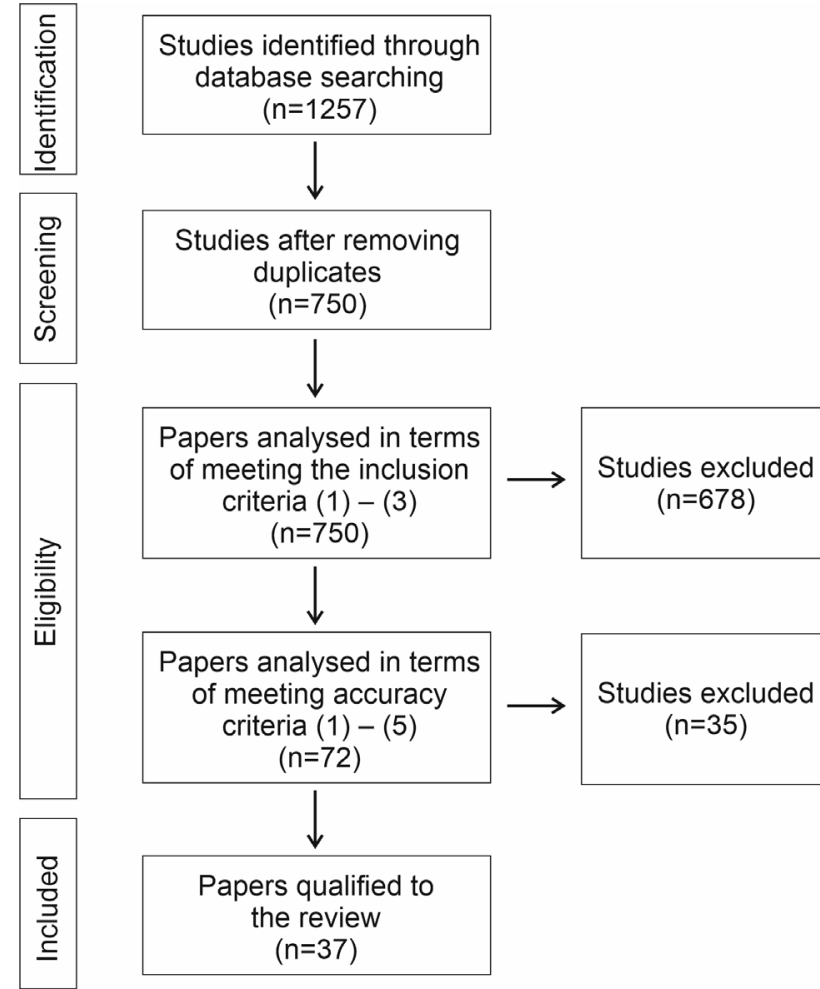

Figure 1. PRISMA flow diagram showing the selection of papers

diseases; (3) publications on the training of personnel who treat/study children with ASD or on the evaluation of their level of knowledge.

\section{Assessment of the quality of included studies}

In order to ensure the accuracy of the review, the first and the second authors independently developed a summary of the included studies. The accuracy of these studies was checked by using a modified checklist with 5 questions as described by Lang et al. [5]: (1) Is there an accurate description of the participants? (2) Is there an accurate description of exercise behaviour being targeted? (3) Is there an accurate summary of the interventional program? (4) Is there an accurate description of outcomes? (5) Is there an accurate summary of the research methodology?

Co-authors analysed the articles in accordance with a checklist, developed their summary, and verified their accuracy. The resulting summaries were discussed, agreed on, and presented in Table 1.

\section{Ethical approval}

The conducted research is not related to either human or animal use.

\section{Results}

A total of 37 publications were selected as a result of the analysis of articles on examining the effect of PA among children with ASD for the period of 2000-2019 (Table 1).

The number of participants involved in the research varied significantly: from 1 person [16-18] or 2-3 people [19-24] up to 112 [25] in 1 group and 58 [26] or 64 [27] individuals in 2 groups. On average, 15 subjects were engaged in the research by specialists (mean [M]: 16.17; standard deviation $[S D]:$ 18.44). The value of the standard error (higher than the arithmetic mean) confirms the wide scope of the number of participants. 


\begin{tabular}{|c|c|c|c|c|c|c|c|c|c|c|c|c|c|c|c|}
\hline & 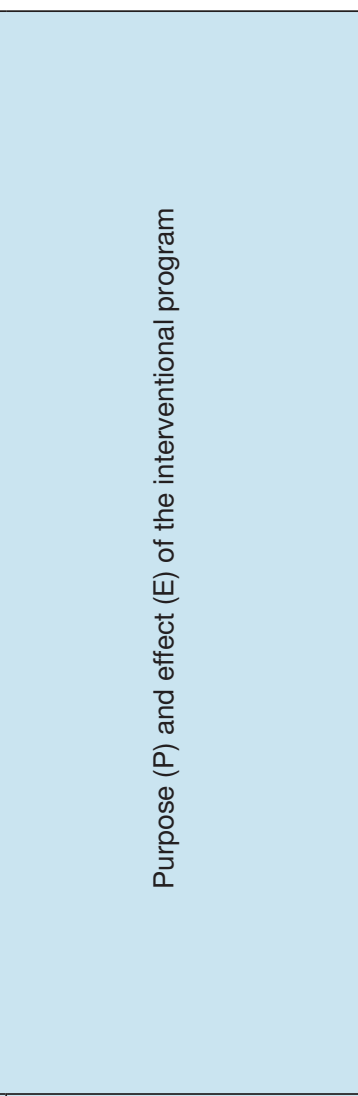 & 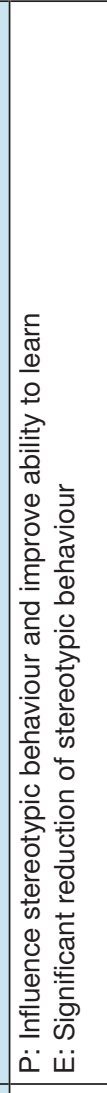 & 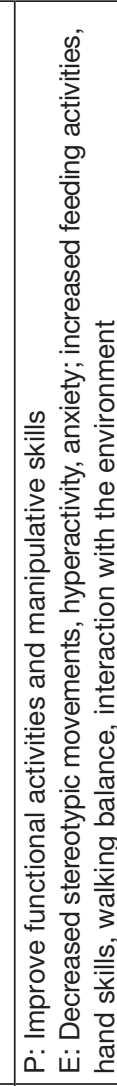 & \multicolumn{2}{|c|}{ 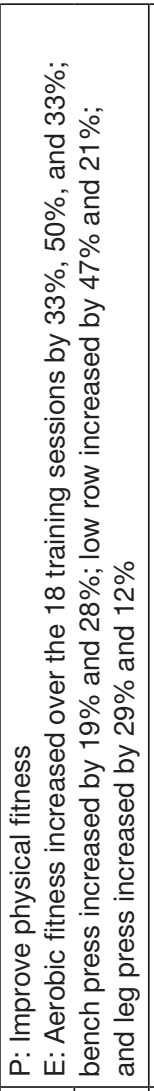 } & 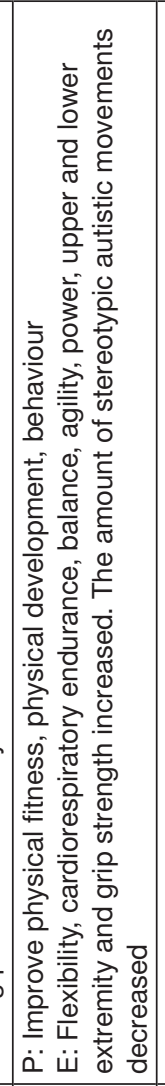 & 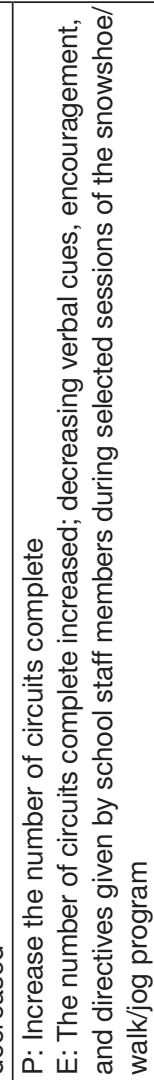 & \multicolumn{2}{|c|}{ 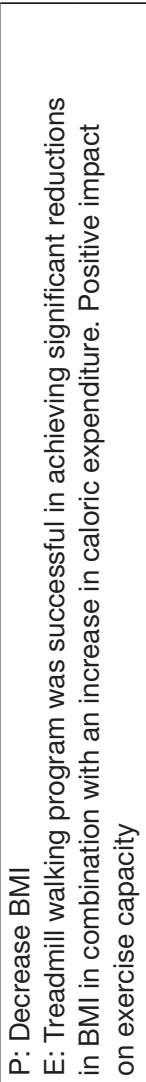 } & \multicolumn{2}{|c|}{ 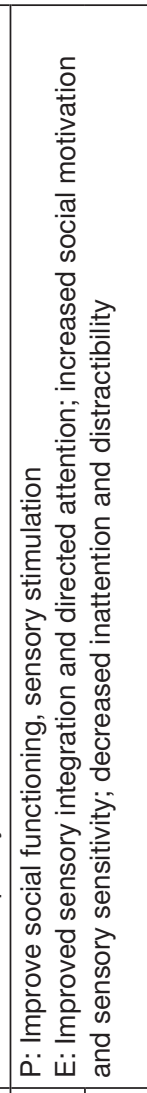 } & \multicolumn{2}{|c|}{ 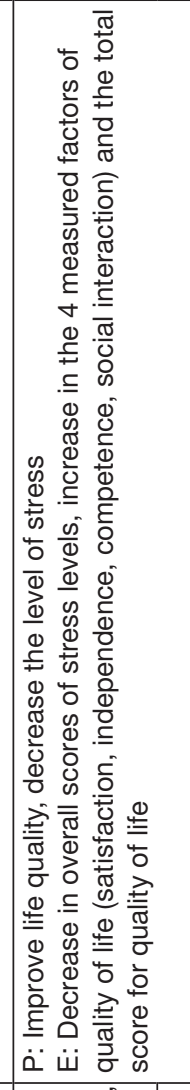 } & \multicolumn{2}{|c|}{ 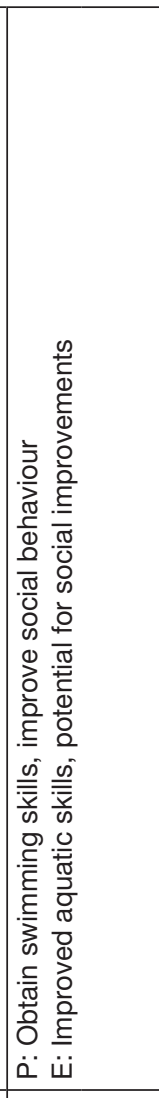 } \\
\hline & $\stackrel{\infty}{\stackrel{2}{\gtrless}}$ & 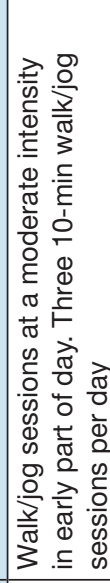 & 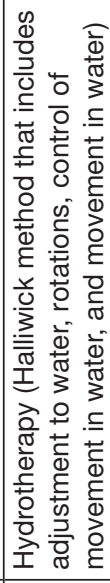 & 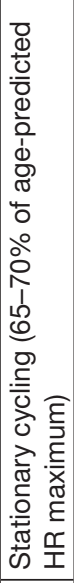 & 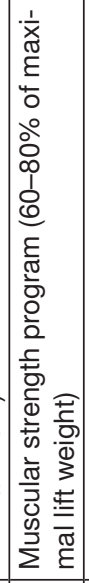 & 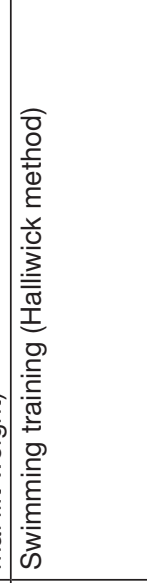 & 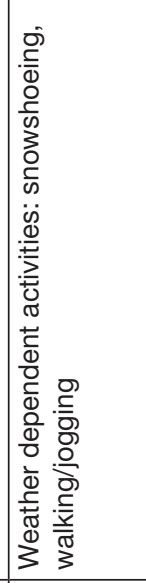 & 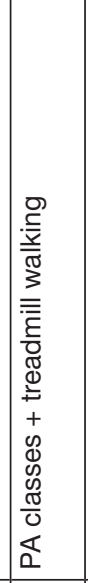 & 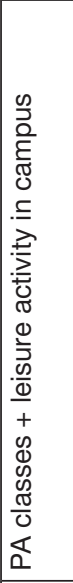 & 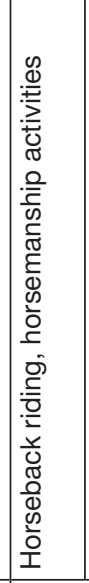 & 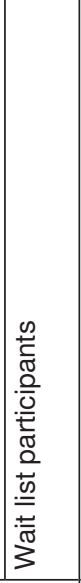 & 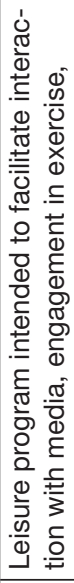 & & 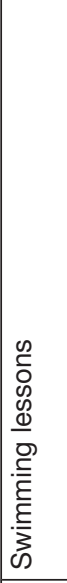 & \\
\hline & (u!щ) әщ!! & 으 & i & i & 8 & 8 & 요 & 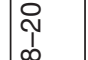 & p & 8 & & $\stackrel{i}{N}$ & । & ৪ & \\
\hline & (yәәм/suo!ssəs) Kouənbəגધ & م & N & $m$ & $\stackrel{m}{a}$ & $m$ & $\sim$ & $\stackrel{L}{p}$ & $m$ & - & & م & 1 & N & \\
\hline & (sуәәм) uo!̣eגn & 立 & $\infty$ & 0 & 0 & 은 & $+\stackrel{\infty}{\infty}$ & 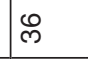 & & $\cong$ & & ธิ & । & *웅우+ & 우웅 \\
\hline & $\begin{array}{l}\frac{0}{0} \\
0 \\
\frac{0}{9} \\
\frac{.0}{0} \\
\frac{0}{0}\end{array}$ & 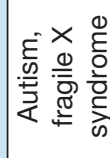 & 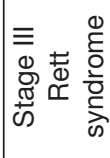 & 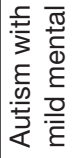 & & 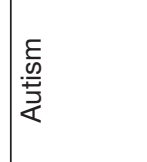 & & 忟 & & 邑 & & 管 & & 量 & \\
\hline & $(\operatorname{sieəК)~әбиел~әб~}$ & $\begin{array}{l}9 \\
1 \\
6\end{array}$ & F & $\begin{array}{l}\bar{t} \\
\underline{\underline{t}}\end{array}$ & & $\infty$ & $\begin{array}{l}\stackrel{\Upsilon}{N} \\
1 \\
\underline{L}\end{array}$ & 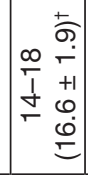 & 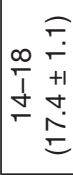 & 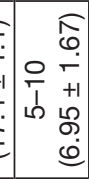 & 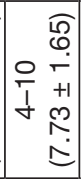 & 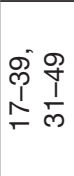 & 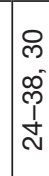 & 早 & \\
\hline & (u) əz! s ə|dures & $\theta$ & $\stackrel{\circ+}{\sim}$ & $m$ & N & - & m & $\begin{array}{l}\text { ot } \\
\text { so } \\
\text { so }\end{array}$ & $\begin{array}{l}\text { iv } \\
\text { so } \\
0\end{array}$ & 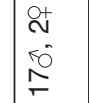 & 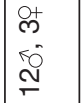 & $\begin{array}{l}\text { if } \\
\text { so } \\
\text { in }\end{array}$ & $\begin{array}{l}8 \\
8 \\
8\end{array}$ & $\infty$ & $\infty$ \\
\hline & sdnoגפ & $\underline{\underline{0}}$ & $\underline{\underline{O}}$ & $\underline{\bar{E}}$ & $\underline{\tilde{E}}$ & $\underline{0}$ & $\underline{0}$ & $\underline{\underline{0}}$ & ग & $\underline{\underline{0}}$ & O & $\underline{\underline{O}}$ & S & $\underline{\bar{J}}$ & $\underline{\underline{\tilde{N}}}$ \\
\hline & $\begin{array}{l}\hat{9} \\
\text { in }\end{array}$ & 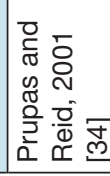 & 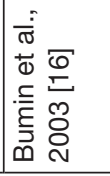 & 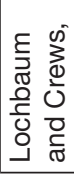 & & 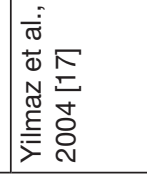 & 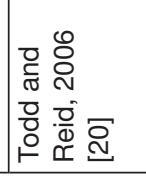 & 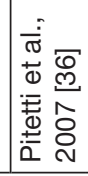 & & 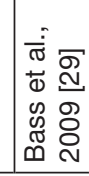 & & 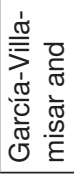 & & S. & \\
\hline
\end{tabular}




\begin{tabular}{|c|c|c|c|c|c|c|c|c|c|c|c|c|c|c|c|}
\hline 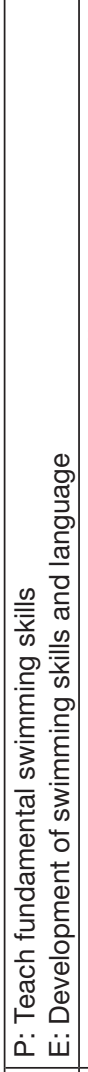 & 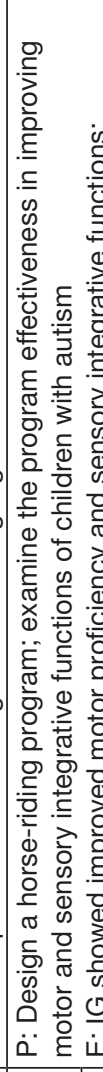 & 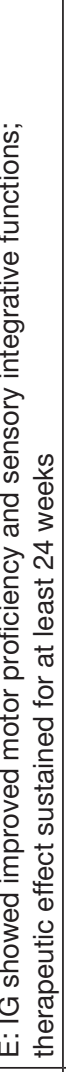 & 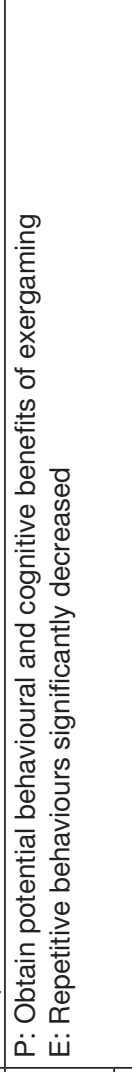 & & 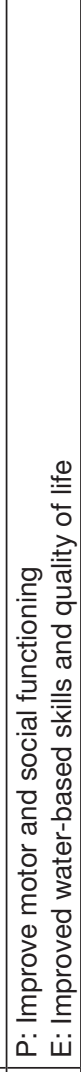 & 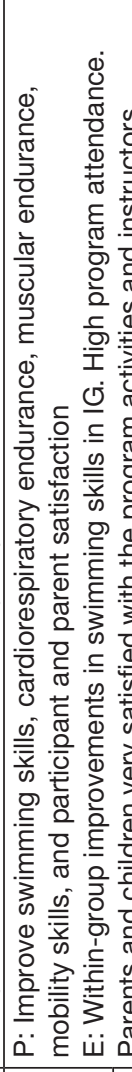 & 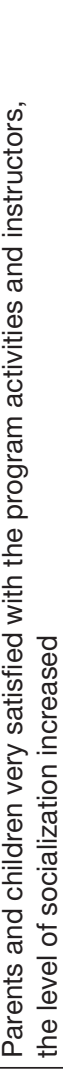 & 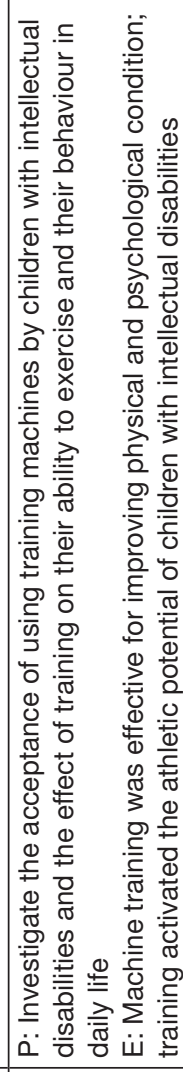 & 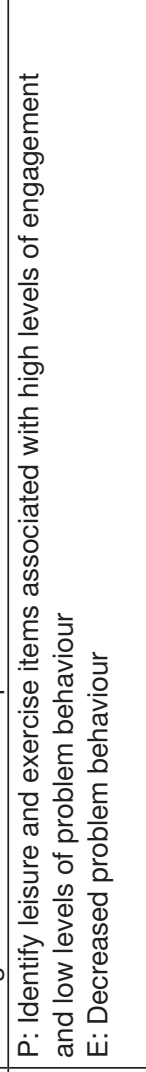 & 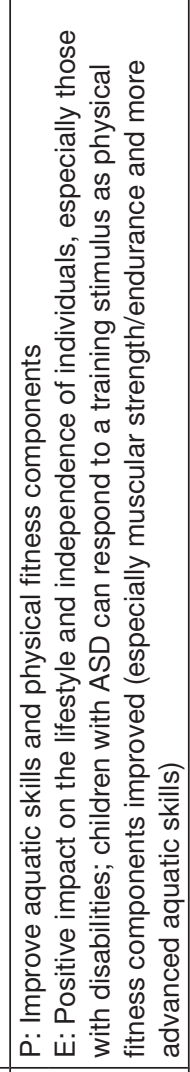 & 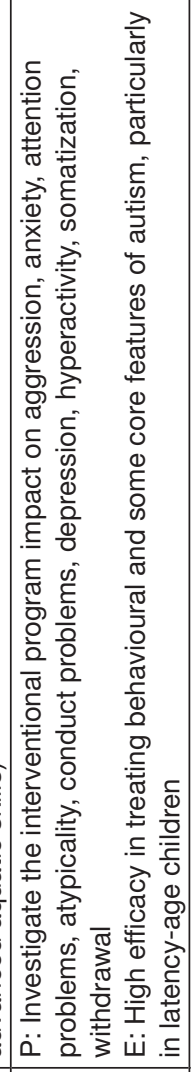 & 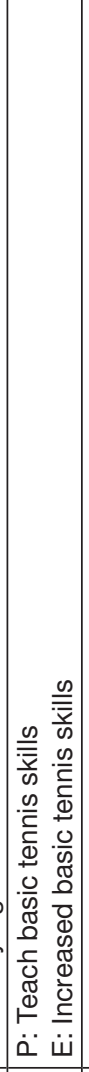 & 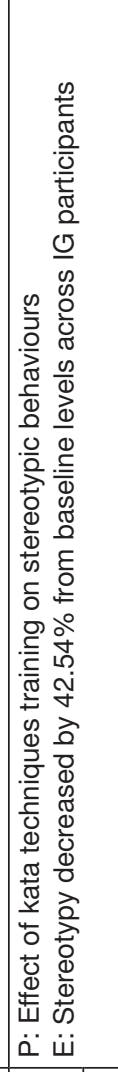 & 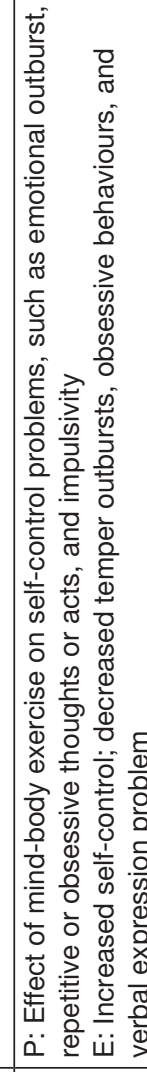 & \\
\hline 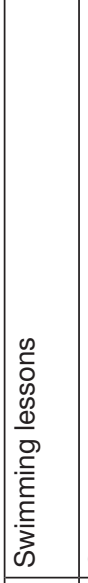 & 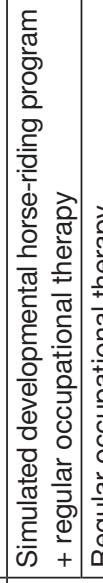 & & 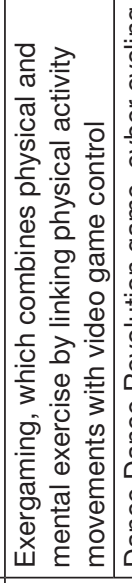 & 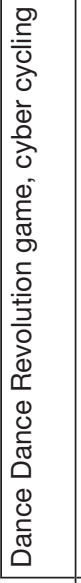 & 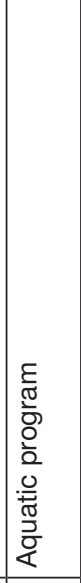 & 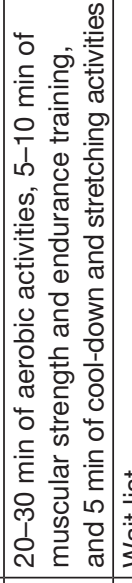 & 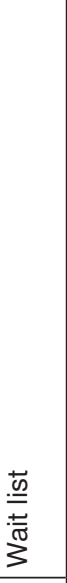 & 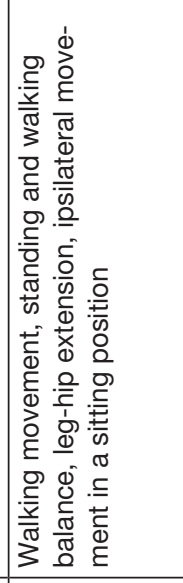 & 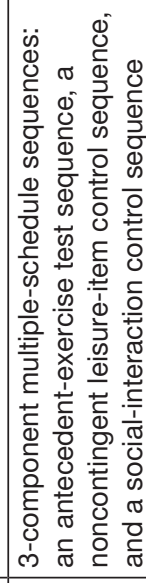 & 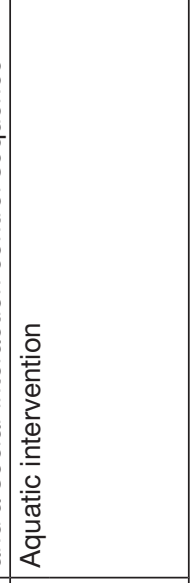 & 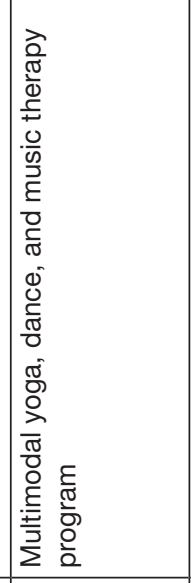 & 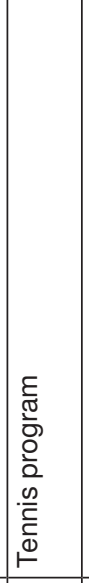 & 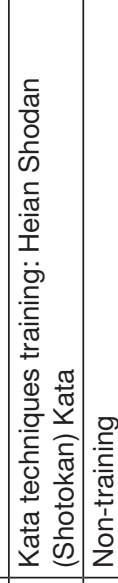 & 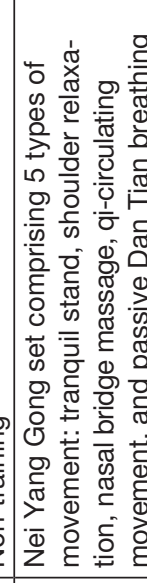 & 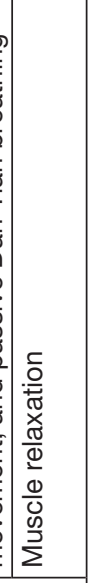 \\
\hline 号 & 8 & & i & & 8 & o & I & ஓ & 只 & 8 & $\stackrel{\text { L }}{q}$ & 8 & | & 8 & \\
\hline Q & $\sim$ & & Q & & $\sim$ & $\sim$ & 1 & - & 之 & N & 之 & م & F & N & \\
\hline i & i & i & $\frac{1}{2}$ & & 움 & I & 1 & $\cong$ & 2 & I & $\infty$ & 0 & I & $\sigma$ & \\
\hline $\begin{array}{l}\frac{E}{0} \\
\frac{D}{\bar{z}} \\
\bar{z}\end{array}$ & 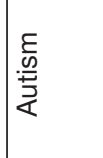 & & कि & & $\begin{array}{l}\frac{E}{D} \\
\frac{D}{5} \\
\frac{D}{\alpha}\end{array}$ & 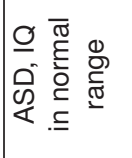 & & $\begin{array}{l}\frac{E}{D} \\
\frac{D}{\bar{z}} \\
\end{array}$ & $\mid$\begin{tabular}{l}
$\frac{E}{D}$ \\
$\frac{D}{\bar{z}}$ \\
\multirow{\alpha}{\alpha}{}
\end{tabular} & 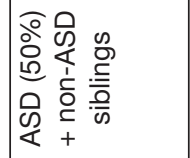 & OP & 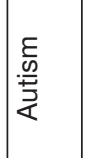 & 要 & 足 & \\
\hline lo & $\mid \begin{array}{l}\infty \\
1 \\
0\end{array}$ & & 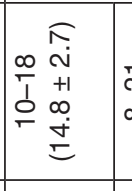 & 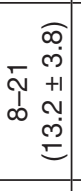 & p) & $\frac{N}{1}$ & & $\begin{array}{l}\infty \\
0 \\
0 \\
+1 \\
0 \\
\dot{0}\end{array}$ & $\begin{array}{l}\overline{\widehat{N}} \\
\hat{\jmath}\end{array}$ & 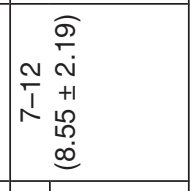 & $\begin{array}{ll}1 & 0 \\
0 & 0 \\
0 & \infty \\
0 & +1 \\
0 & 0 \\
\infty & 0 \\
\infty & 0\end{array}$ & 早 & 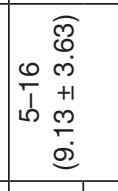 & 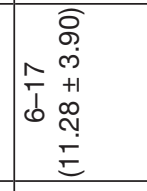 & 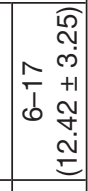 \\
\hline$m$ & পি & ले & $\cong$ & 우 & $\frac{+}{6}$ & $\cong$ & \llcorner & N & $\begin{array}{l}0+ \\
N \\
\text { so } \\
N\end{array}$ & $\forall \stackrel{\square}{\square}$ & 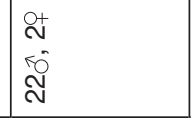 & 每 & 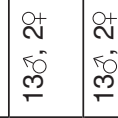 & ) & 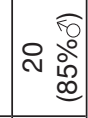 \\
\hline$\underline{0}$ & $\underline{0}$ & S & $\overline{\bar{J}}$ & $\underline{\tilde{N}}$ & $\underline{\sigma}$ & $\underline{0}$ & S & $\underline{0}$ & $\underline{0}$ & $\underline{0}$ & $\underline{0}$ & $\underline{0}$ & ک ত S & $\underline{0}$ & O \\
\hline 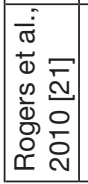 & 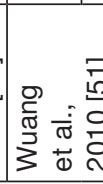 & & 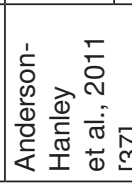 & & 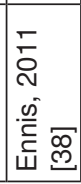 & 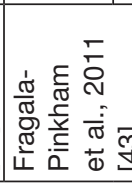 & & 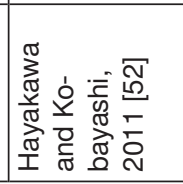 & 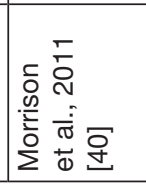 & 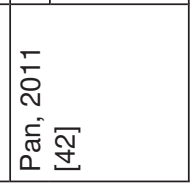 & 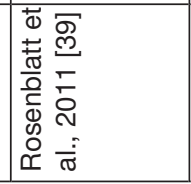 & 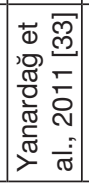 & 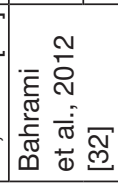 & 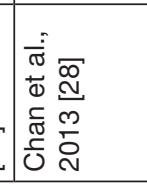 & \\
\hline
\end{tabular}




\begin{tabular}{|c|c|c|c|c|c|c|c|c|c|c|c|c|c|c|c|c|c|c|}
\hline 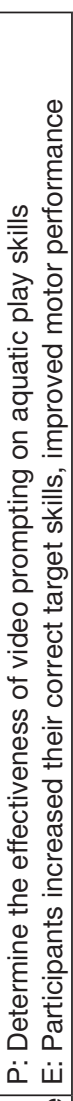 & 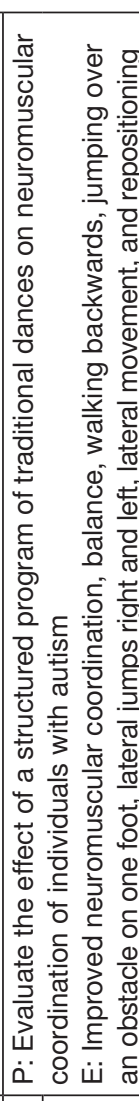 & 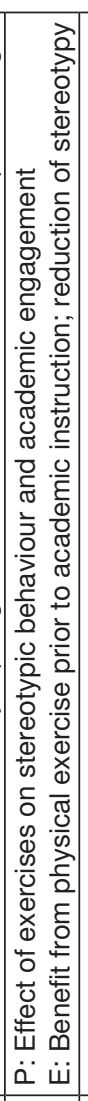 & 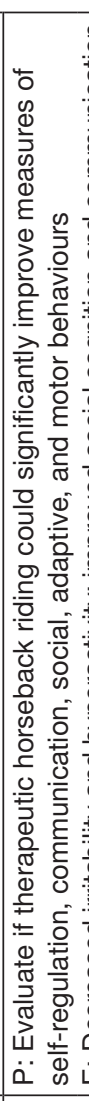 & 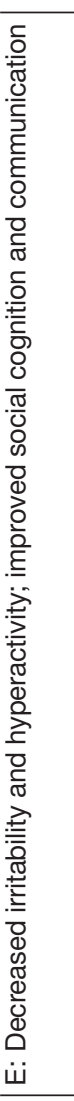 & 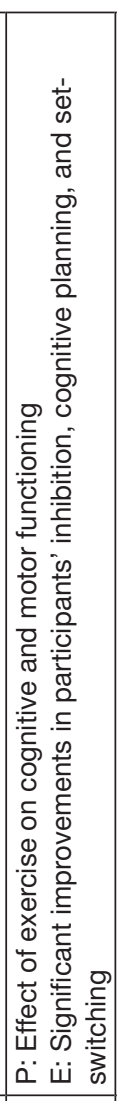 & 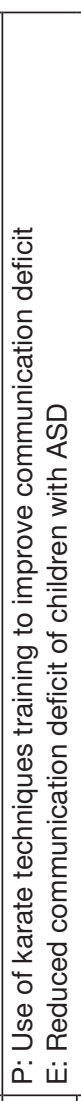 & & & 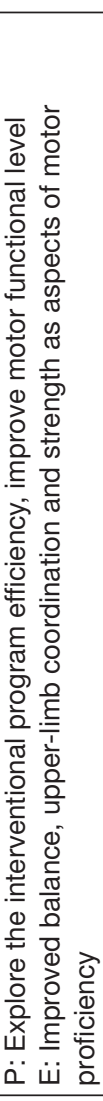 & 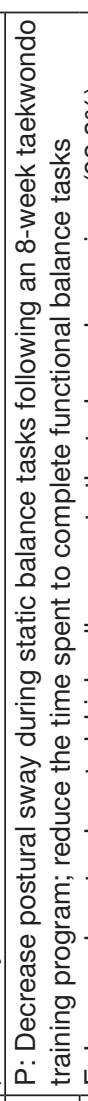 & 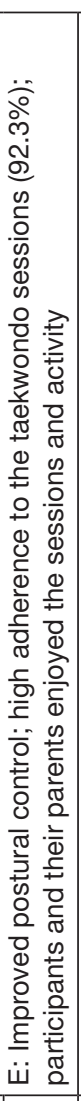 & 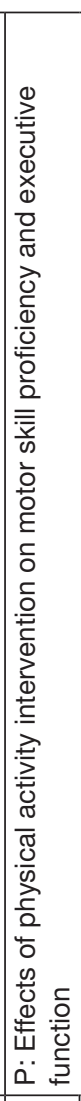 & 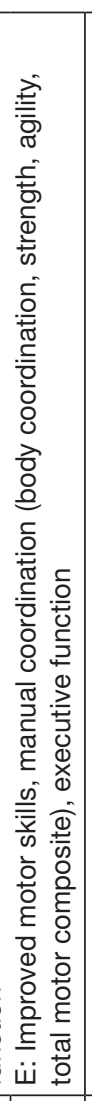 & 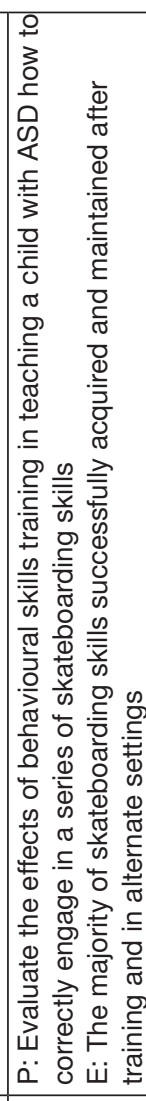 & 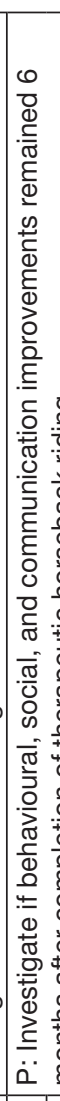 & 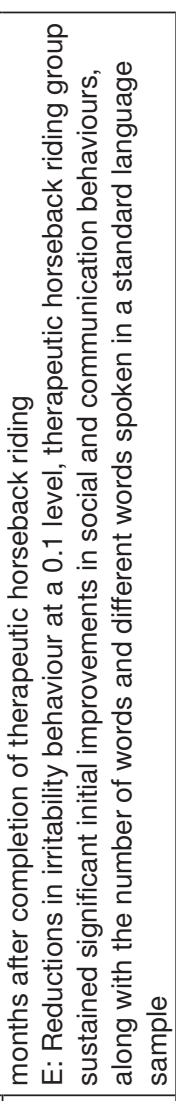 & 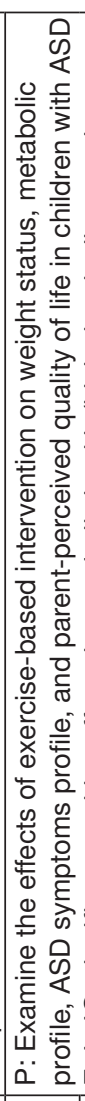 & 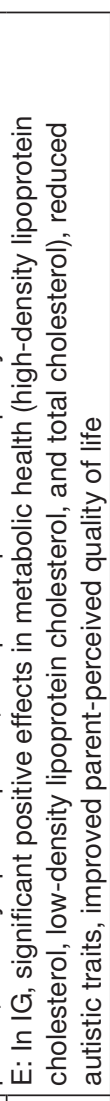 \\
\hline 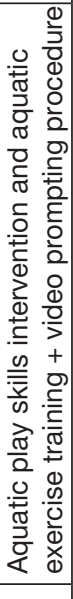 & 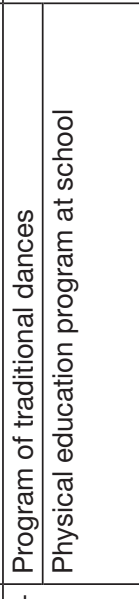 & 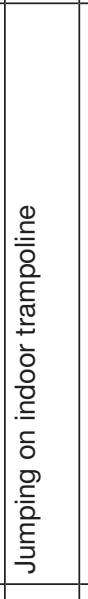 & 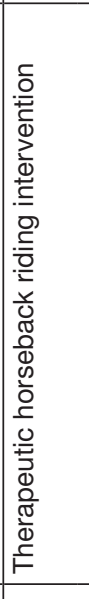 & & 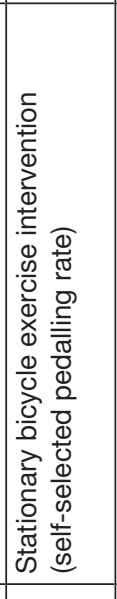 & 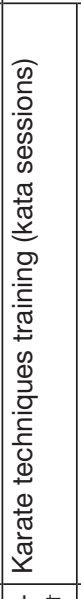 & 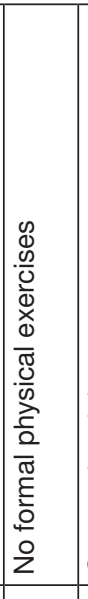 & & 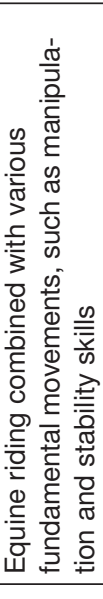 & 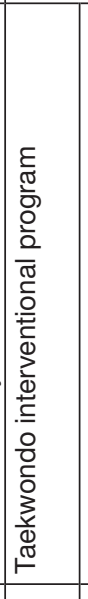 & 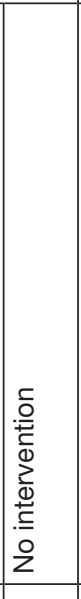 & 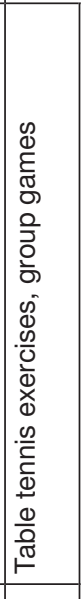 & 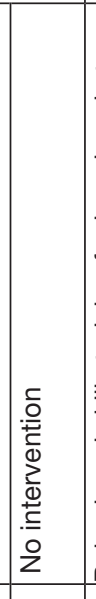 & 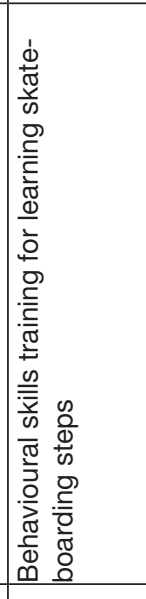 & 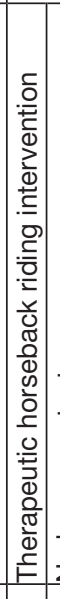 & 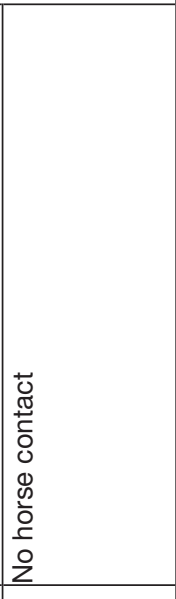 & 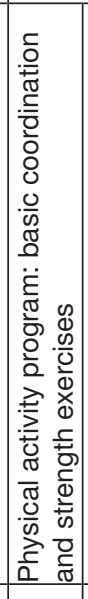 & 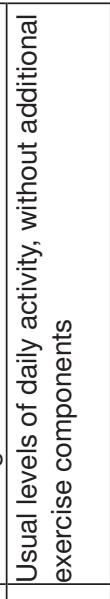 \\
\hline 8 & 占 占 & 우 & 过 & & i & 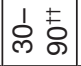 & 1 & 1 & లి & in & 1 & i & 1 & i & 过 & 1 & Io & 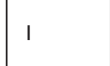 \\
\hline$m$ & $m$ & $\begin{array}{l}m \\
\stackrel{1}{N} \\
N\end{array}$ & z & & Z & $\theta$ & 1 & 1 & - & $\sim$ & 1 & N & 1 & 只 & 吕 & 1 & $\sim$ & 1 \\
\hline$\stackrel{N}{\sim}$ & $\infty$ & $\underset{1}{1} \simeq$ & 으 & & Z & I & 1 & & 으 & $\infty$ & 1 & $\cong$ & 1 & 只 & 웅 & 1 & $\underset{q}{\infty}$ & 1 \\
\hline 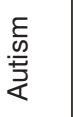 & 竞 & 角 & 总 & & 总 & 亘 & & & 足 & कि & & कि & & 旁 & 曽 & & & \\
\hline $\begin{array}{l}\infty \\
1 \\
0\end{array}$ & 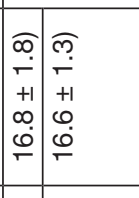 & $\underset{p}{\infty}$ & 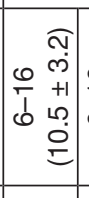 & 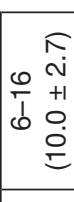 & 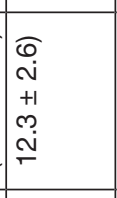 & $\begin{array}{|cc|} & \widehat{N} \\
0 & \mathbf{m} \\
0 & 0 \\
1 & +1 \\
0 & 0 \\
0 \\
0 \\
0\end{array}$ & $\mid$\begin{tabular}{rr|} 
& $\widehat{m}$ \\
0 & 0 \\
0 & 0 \\
1 & +1 \\
0 & 0 \\
0 \\
0
\end{tabular} & & $\begin{array}{l}\infty \\
\sigma^{\infty}\end{array}$ & 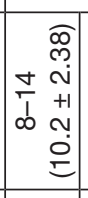 & 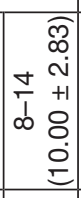 & 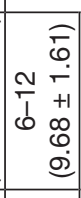 & 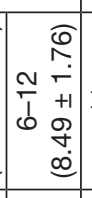 & $F$ & $\begin{array}{l}0 \\
1 \\
1 \\
0\end{array}$ & & $\mid \begin{array}{l}N \\
1 \\
0\end{array}$ & No \\
\hline 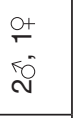 & \begin{tabular}{|l|l|l|l|l|l|l|l} 
& 0
\end{tabular} & 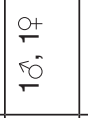 & 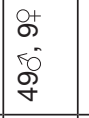 & $\begin{array}{l}8+ \\
0 \\
i 0 \\
\text { î } \\
\end{array}$ & $\begin{array}{l}\text { if } \\
\text { in } \\
\text { in } \\
\end{array}$ & 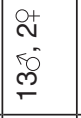 & 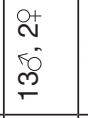 & $\mp$ & $\begin{array}{l}\text { so } \\
\text { of } \\
\text { of }\end{array}$ & $\infty$ & $\begin{array}{l}\text { O+ } \\
\stackrel{+}{50} \\
\text { in } \\
\text { in }\end{array}$ & $\stackrel{F}{F}$ & $\stackrel{\circ}{\mp}$ & 50 & & $\stackrel{\infty}{\sim}$ & \& & $\stackrel{\infty}{\sim}$ \\
\hline$\underline{\underline{0}}$ & \begin{tabular}{|l|l}
$\underline{0}$ & 0 \\
\end{tabular} & $\underline{\underline{0}}$ & $\underline{\underline{\sigma}}$ & J & $\underline{\underline{\sigma}}$ & $\underline{0}$ & J & \begin{tabular}{|l|} 
\\
\end{tabular} & $\underline{\underline{0}}$ & $\underline{0}$ & S & $\underline{0}$ & O & $\underline{\underline{0}}$ & & O & 으 & 0 \\
\hline 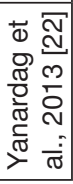 & 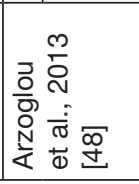 & 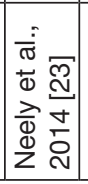 & 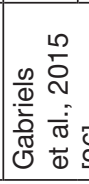 & & 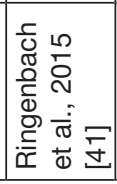 & & & & 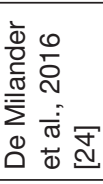 & 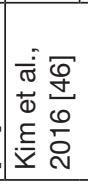 & & 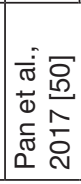 & & 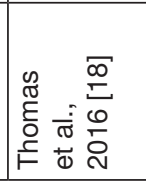 & 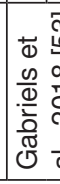 & 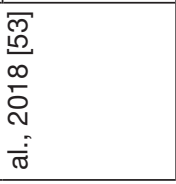 & & \\
\hline
\end{tabular}




\begin{tabular}{|c|c|c|c|c|c|c|c|}
\hline \multicolumn{2}{|l|}{ 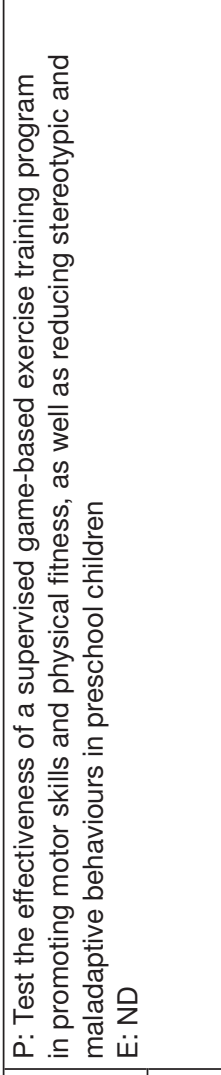 } & \multicolumn{2}{|c|}{ 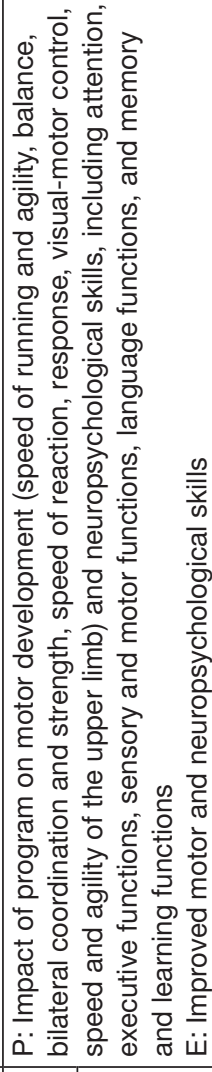 } & \multicolumn{2}{|l|}{ 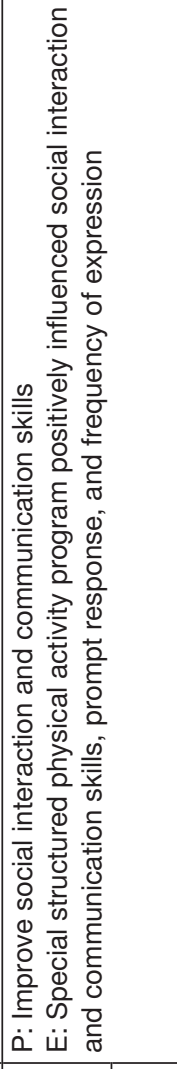 } & 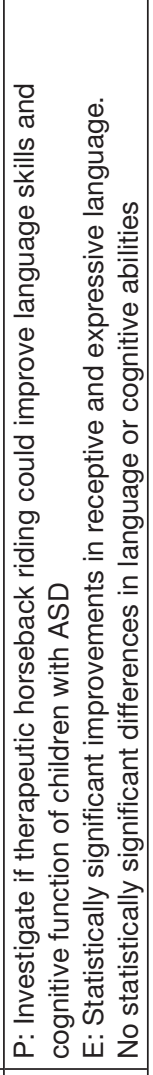 & 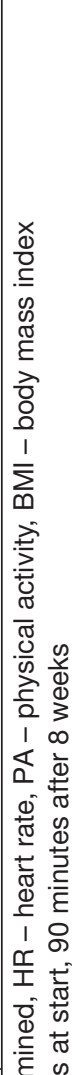 \\
\hline 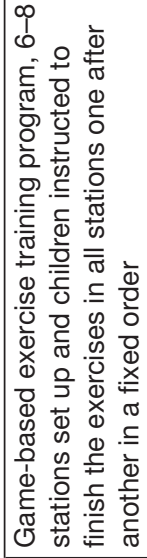 & 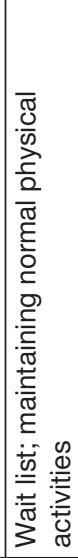 & 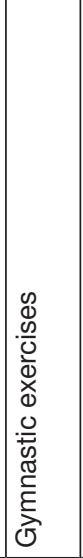 & 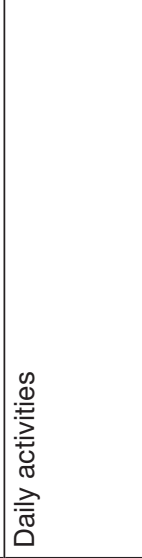 & 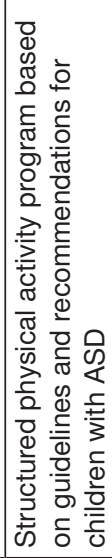 & 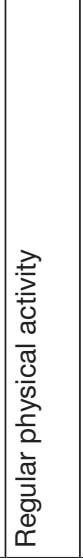 & 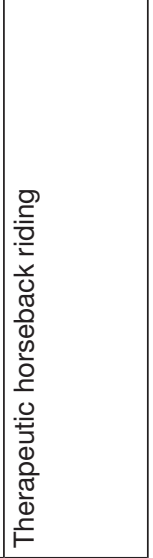 & 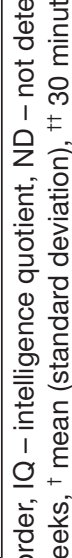 \\
\hline \multicolumn{2}{|l|}{ ○ } & \multicolumn{2}{|l|}{$\mathscr{q}$} & \multicolumn{2}{|l|}{8} & ㅇ & \\
\hline \multicolumn{2}{|l|}{$\sim$} & \multicolumn{2}{|l|}{$m$} & \multicolumn{2}{|l|}{$\sim$} & - & \\
\hline \multicolumn{2}{|l|}{$\stackrel{0}{ }$} & \multicolumn{2}{|l|}{$\mathscr{\varphi}$} & \multicolumn{2}{|l|}{ 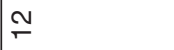 } & $\infty$ & \\
\hline \multicolumn{2}{|l|}{$\stackrel{0}{<}$} & \multicolumn{2}{|l|}{ 足 } & \multicolumn{2}{|l|}{ 安 } & 足 & 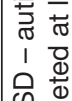 \\
\hline \multicolumn{2}{|l|}{$\frac{1}{t}$} & 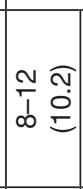 & 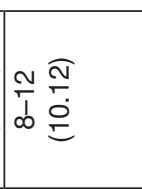 & 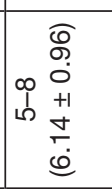 & 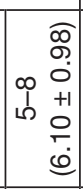 & $\frac{m}{1}$ & 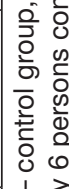 \\
\hline \multicolumn{2}{|l|}{$\frac{7}{7}$} & $\begin{array}{l}\mathrm{P}+ \\
\mathrm{r} \\
\mathrm{s}^{2} \\
\infty\end{array}$ & 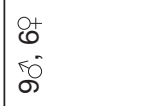 & 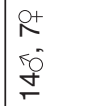 & $\begin{array}{l}\text { if } \\
\text { in } \\
50 \\
10 \\
0\end{array}$ & $\stackrel{\infty}{\leftarrow}$ & + \\
\hline$\underline{\underline{\sigma}}$ & U & $\underline{\sigma}$ & O & $\underline{\underline{\sigma}}$ & O & $\underline{0}$ & \\
\hline \multicolumn{2}{|l|}{ 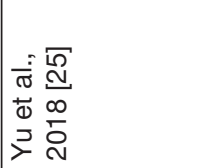 } & \multicolumn{2}{|c|}{ 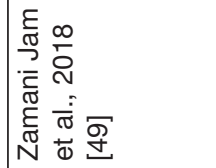 } & \multicolumn{2}{|l|}{ 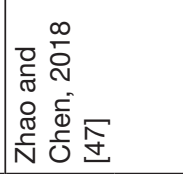 } & 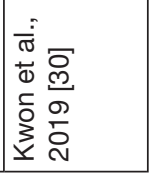 & 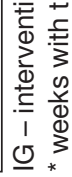 \\
\hline
\end{tabular}

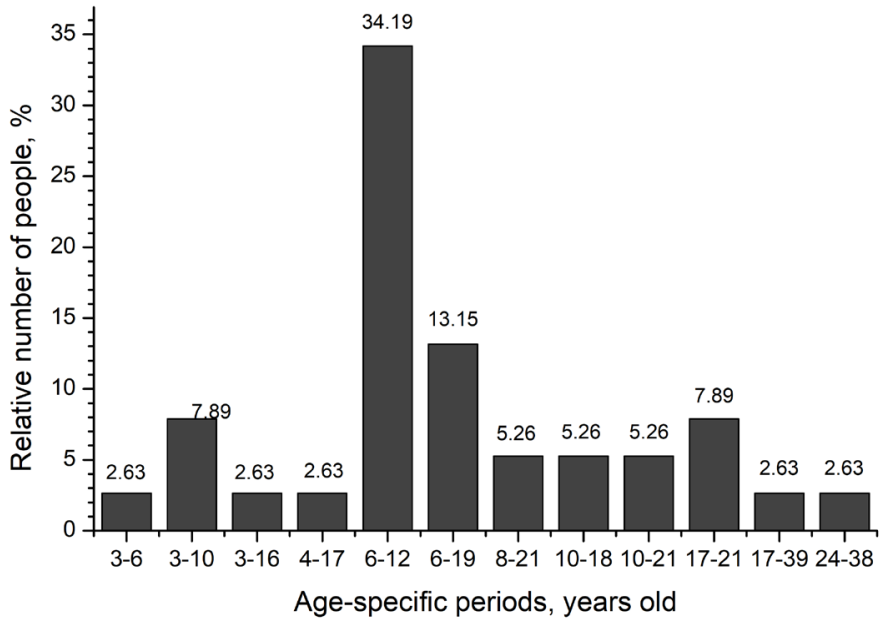

Figure 2. Proportions (\%) of subjects with autism spectrum disorders of different age ranges in the included studies $(n=37)$

The contingent of test subjects (84.2\%) usually consisted of school-age children. In every third case (34.2\%), children aged 6-12 years were involved in the research (Figure 2). The age of the participants ranged from 3 to 39 years; quite often, heterogeneous age groups were formed. Individuals of different ages were frequently engaged in one group of the PE program; in particular, $57.9 \%$ of the articles concerned children of primary, secondary, and senior school age, as well as adults.

The duration of PE programs ranged from 4 [28] up to 48 weeks [27, 28] and equalled 13.55 (SD: 10.70) weeks on average. The duration of the main part of the analysed programs (63.2\%) did not exceed 8-14 weeks (M: 10 weeks).

The frequency of training in experimental programs amounted to 2.57 (SD: 1.14) times/week, but also has fluctuated significantly - from 1 time/week [24, 29, 30] up to 4-5 times/week (kata studying, cycling [31, 32], tennis lessons [33], walking in alternation with jogging [34], outdoor activities [35]).

In $21 \%$ of the analysed works, the duration of the training lessons was not indicated; however, in accordance with the information available, it can be concluded that usually they lasted for 45-60 minutes ( $M$ : $50.20 \mathrm{~min}$; $S D$ : $21.18 \mathrm{~min}$; median: $60 \mathrm{~min}$; maximum: $120 \mathrm{~min}$; minimum: $10 \mathrm{~min}$ ). The duration of a lesson with a stationary bike, walking on a treadmill, walking in alternation with jogging, exergaming sessions did not exceed 20 minutes [19, 34, 36, 37]. In rare cases, e.g. training in karate technique, the sessions lasted up to 1.5 hours [31, 32]. Recreational classes were 2 hours long [29].

Few interventional programs [17, 18, 20-25, 33, 38-41] did not involve a control group, which allows to consider them insufficiently substantiated. There is practically no information on the intensity of the physical load applied, the intelligence quotient (IQ) level of children involved in the examination, the volume of PA, or the level of the participants' functional capabilities.

A significant part of the works studied the effects of water exercises [16, 22, 38, 42, 43]. It was proved that hydrotherapy had a positive effect on the technical skills of people with ASD (e.g. on the formation of new skills [42-44]) and increased physical fitness levels (in particular, leg and hand strength, flexibility, cardiorespiratory endurance, balancing, and dexterity $[42,43])$. Water exercises exerted a positive impact on the behaviour of ASD individuals and their interaction with others [34, 36]; a decrease in hyperactivity, anxiety, and stereotypy was also observed $[34,42,44]$. Physical exercises 
in water positively influenced various indicators of quality of life, in particular, those of physical, emotional, social, and school functioning.

Hippotherapy training courses [26, 29] turned out useful to improve motor skills, reduce the number of stereotypies and mood disorders, increase self-regulation and socialization [26], strengthen social motivation, reduce sensory sensitivity, overcome and correct absent-mindedness, increase concentration, boost fine motor skills [26, 29], as well as improve communication skills [26] of children with ASD.

The use of yoga and martial arts had a positive effect on stereotypic behaviour [32, 33], overcoming difficulties with communication [31, 33], interaction with others [39, 45], improving some physical fitness indicators (enhancing balance while standing on one and two legs) [46].

Cycling positively affected repetitive behaviour, cognitive functions, attentiveness, switching, perception, suppression, and executive functions [37, 41]; it also enhanced cognitive planning [42].

Positive effects were observed under the influence of exercises that required complex coordination. So, the researchers found out a positive impact of skateboarding on the formation of new motor skills [18]. Scientists proved positive results of dancing on repetitive behaviour, cognitive functions, executive function [37], behavioural problems [39], physical fitness, and motor skills $[47,48]$ of children with ASD. The effect of gymnastics exercises on self-control [28, 49], speech development [49], and physical fitness indicators [49] were also established.

Exercise programs involving cardio and strength fitness significantly increased the level of physical fitness in children with ASD; they primarily improved aerobic endurance and muscle strength [19]. The use of exergaming reduced the number of actions with stereotypic behaviour, improved cognitive and executive functions of children with ASD [37]. The application of outdoor games, as well as training programs with the priority usage of elements of sports games increased PA [50] and positively affected the motor abilities of children with ASD: hand and body coordination, strength and dexterity [22, 33, 50]; it also improved executive function [22].

\section{Discussion}

The results of the analysis showed that in years 20002019 , the number of studies on the effect of PE classes in children with ASD gradually increased. This is a consequence of a real rise in researchers' interest in this topic, since the use of physical exercises for therapeutic purposes is economically advantageous, has no contraindications [54-56], and comprehensively affects a wide range of indicators, including those reflecting the main development problems of children with ASD.

Despite the significant amount of scientific research held over the past 20 years, evidence of the positive effect of PA on overcoming manifestations of problematic behaviour and improving the psycho-functional state of children with ASD remains limited [57-60]. In the period of 2000-2019, most of the studies were conducted for the age group of 6-21 years. Our data indicate a wide range of ages of participants in pedagogical experiments, and thus confirm the results of other researchers who noted that the age of experiment subjects ranged from 4 to 27 years [13] and from 3 to 41 years [5]. Owing to the heterogeneity of the contingent, the conclusions of most scientific articles should be clarified.
At the same time, there is a lack of scientific research that would study various groups of indicators (physical, cognitive, and indicators of problematic areas in children with ASD: behavioural and communicative ones) as a whole. This does not allow finding out the leading ones for accented development.

It is worth noting that the existing studies were often heterogeneous, not only in terms of the participants' age but also with regard to their IQ levels, the volume of their daily PA, and their functional capabilities. Because of the limited overall number of children with ASD, scientists often combine investigations of children of related age ranges into a single group, which is unacceptable for research among children without ASD. Also, the subjects' IQ level was often not indicated, although such information is essential in interpreting numerous indicators. For example, more than $50 \%$ of children with ASD may achieve poor results during testing, not as a result of insufficient levels of PA, but because of inconsistencies or lack of understanding of the instructions [61, 62]. Authors usually do not indicate the level of children's functional capabilities, although there is information on large differences in the volume of PA [13, 63] among children with ASD and the related capabilities of such children, which can lead to significant functional differences. Children with ASD can significantly lag behind their peers in many other indicators, too [8, 25, 64-66]. Therefore, in research results analyses, they must be clustered to form more homogeneous samples [67-69].

Unfortunately, detailed information on the specifics of interventions for children with ASD, such as training intensity, volume, and frequency, was also mostly absent in publications for the period of 2000-2019. The intensity of the physical load was referred to only in individual studies, which does not allow exactly reproducing the training programs in practice and achieving the desired effect.

According to our data, PA programs lasted 4-48 weeks, but in most cases (60\%) they were short-term and did not exceed 8-14 weeks. Often, the delayed effects were not studied. It was found that the frequency of intervention was 3 times/week on average, with sessions lasting for 60 minutes (minimum of 10 minutes, up to 120 minutes as maximum). This partially confirms the results described in specialist literature that the duration of interventions varies from 8 to 36 weeks, with a frequency of training classes of 2-3 times/ week and the duration of the exercise sessions of 20-40 minutes [70]. So, training classes held 3 times/week for 4560 minutes each, applied for 8 weeks within a program that provides for an integrated use of PE, can have a significant effect.

There were some reservations that the duration of positive effects after physical exercises was estimated by specialists infrequently and in small samples. Indeed, the positive effects of applying physical exercises may be temporary [5]. In such cases, in order to consolidate the effect, experts [34] have recommended conducting several physical exercises a day.

The results of our analysis confirm the observations from numerous studies on the effectiveness of water exercises, as well as running or walking [15]. However, for the first time, we proved the increased interest of researchers in studying the effectiveness of hippotherapy and martial arts for solving the problems of children with ASD.

The analysis of the obtained data shows that most researchers studied the influence of PE programs using one type of PA. Only some of them concerned verifying the effectiveness of comprehensive programs that used a wide 
range of tools. For example, we examined the effectiveness of a relaxation exercise program with dance and music therapy tools [39], programs containing various types of exercises [40], training programs involving coordination and strength exercises [27], structured PA programs with naturally integrated elements of social interaction [53]. Some studies compared the effectiveness of two different programs: e.g. weight training and cardio fitness [19], yoga and Chinese gymnastics [28], one-time or three-time jogging loads [34], dance and exercise bike exergaming [37], or even three training programs: assistive bicycle therapy, cycling, and overall absence of cyclic applications [41].

The obtained results indicate that scientists usually studied only 1 or 2 indicators of 1 , rarely 2 sets (physical, cognitive, and indicators of problem areas of children with ASD: behavioural and communicative ones), while there are only a few complex multidisciplinary studies in the area. The frequency of studies of communicative and cognitive indicators sets $(12 \%$ and $10 \%$, respectively) significantly $(p<0.05)$ lags behind the frequency of studies of physical and behavioural indicators in children with ASD $35 \%$ and $31 \%$, respectively). The popularity of studying these indicators is associated with the high social significance of such treatment goals and the critical importance of reducing harmful behaviour [13]. Our results are in line with data [13] stating that the most significant effect is observed by scientists in terms of physical development and physical fitness, and slightly less substantial results are found with reference to stereotypic and self-harming behaviour. Low evidence of PA exposure was noted in cognitive performance for adolescents, executive function (e.g. working memory, cognitive control, and attention), as well as better behavioural control (e.g. reduced aggression and disruptive behaviour) [13].

Data on changes in quality of life (4\%), the volume of PA (3\%), and body weight (4\%) in children with ASD are scarce and contradictory. At the same time, there is a lack of scientific research that would study different sets of indicators in an integrated manner.

\section{Conclusions}

1. The scientific research in the period of 2000-2019 devoted to the shifting effect of applied PE programs for children with autism usually included an age-heterogeneous contingent, corresponding to the level of children's functionality and their IQ level. The number of individuals involved in the research varied significantly (from 1 person up to 112). The population of test subjects $(84.2 \%)$ usually consisted of school-age children; in every third case, children aged 6-12 years took part in the research.

2. The duration of the experiments was small: up to 8-14 weeks; the frequency of interventions differed, but usually equalled 3 times/week. Classes lasted for 45-60 minutes (but their duration varied greatly: from 10 up to 120 minutes). Therefore, it can be assumed that an experimental program could have a significant effect with a duration of 8 weeks and with training classes held 3 times/week, with several exercises per day lasting 45-60 minutes each, and a clearly fixed volume and intensity of the program involving complex application of PE means with proven efficiency (swimming and playing in water, running, walking, as well as imitation of riding, elements of yoga and oriental martial arts).

3 . It is worth conducting a randomized study with a representative sample, among a contingent of the same age, IQ level, functional capacity, and daily PA application. The study should preferably include indicators of all groups (physi- cal, cognitive, behavioural, and communicative ones) in an integrated manner, paying particular attention to those that are of interest for practice (quality of life, body weight, etc.) but have been infrequently investigated. The duration of the positive effects of physical exercise should be evidenced.

\section{Disclosure statement}

No author has any financial interest or received any financial benefit from this research.

\section{Conflict of interest}

The authors state no conflict of interest.

\section{References}

1. Bourke J, de Klerk N, Smith T, Leonard H. Populationbased prevalence of intellectual disability and autism spectrum disorders in Western Australia: a comparison with previous estimates. Medicine. 2016;95(21):e3737; doi: 10.1097/MD.0000000000003737.

2. Payakachat N, Tilford JM, Kovacs E, Kuhlthau K. Autism spectrum disorders: a review of measures for clinical, health services and cost-effectiveness applications. Expert Rev Pharmacoecon Outcomes Res. 2012;12(4): 485-503; doi: 10.1586/erp.12.29.

3. Autism and Developmental Disabilities Monitoring Network Surveillance Year 2002 Principal Investigators, Centers for Disease Control and Prevention. Prevalence of autism spectrum disorders - autism and developmental disabilities monitoring network, 14 sites, United States, 2002. MMWR Surveill Summ. 2007;56(1):12-28.

4. Kim YS, Leventhal BL, Koh YJ, Fombonne E, Laska E, Lim EC, et al. Prevalence of autism spectrum disorders in a total population sample. Am J Psychiatry. 2011;168(9): 904-912; doi: 10.1176/appi.ajp.2011.10101532.

5. Lang R, Koegel LK, Ashbaugh K, Regester A, Ence W, Smith W. Physical exercise and individuals with autism spectrum disorders: a systematic review. Res Autism Spectr Disord. 2010;4(4):565-576; doi: 10.1016/j.rasd. 2010.01.006.

6. Must A, Phillips SM, Curtin C, Anderson SE, Maslin M, Lividini $\mathrm{K}$, et al. Comparison of sedentary behaviors between children with autism spectrum disorders and typically developing children. Autism. 2014;18(4):376-384; doi: 10.1177/1362361313479039.

7. Tyler K, MacDonald M, Menear K. Physical activity and physical fitness of school-aged children and youth with autism spectrum disorders. Autism Res Treat. 2014;2014: 312163; doi: 10.1155/2014/312163.

8. Jansiewicz EM, Goldberg MC, Newschaffer CJ, Denckla MG, Landa R, Mostofsky SH. Motor signs distinguish children with high functioning autism and Asperger's syndrome from controls. J Autism Dev Disord. 2006;36(5): 613-621; doi: 10.1007/s10803-006-0109-y.

9. Baranek GT, Parham LD, Bodfish JW. Sensory and motor features in autism: assessment and intervention. In: Volkmar FR, Paul R, Klin A, Cohen D (eds.), Handbook of autism and pervasive developmental disorders. Hoboken: John Wiley \& Sons; 2005; 831-857.

10. Ament K, Mejia A, Buhlman R, Erklin S, Caffo B, Mostofsky S, et al. Evidence for specificity of motor impairments in catching and balance in children with autism. J Autism Dev Disord. 2015;45(3):742-751; doi: 10.1007/ s10803-014-2229-0.

11. Todd T. Teaching motor skills to individuals with autism spectrum disorders. J Phys Educ Recreat Dance. 2012; 83(8):32-48; doi: 10.1080/07303084.2012.10598827. 
12. Tan BWZ, Pooley JA, Speelman CP. A meta-analytic review of the efficacy of physical exercise interventions on cognition in individuals with autism spectrum disorder and ADHD. J Autism Dev Disord. 2016;46(9):31263143; doi: 10.1007/s10803-016-2854-x.

13. Sorensen C, Zarrett N. Benefits of physical activity for adolescents with autism spectrum disorders: a comprehensive review. Rev J Autism Dev Disord. 2014;1(4): 344-353; doi: 10.1007/s40489-014-0027-4.

14. Healy S, Nacario A, Braithwaite RE, Hopper C. The effect of physical activity interventions on youth with autism spectrum disorder: a meta-analysis. Autism Res. 2018; 11(6):818-833; doi: 10.1002/aur.1955.

15. Sowa M, Meulenbroek R. Effects of physical exercise on autism spectrum disorders: a meta-analysis. Res Autism Spectr Disord. 2012;6(1):46-57; doi: 10.1016/j.rasd.2011. 09.001.

16. Bumin G, Uyanik M, Yilmaz I, Kayihan H, Topcu M. Hydrotherapy for Rett syndrome. J Rehabil Med. 2003;35(1): 44-45; doi: 10.1080/16501970306107.

17. Yilmaz I, Yanardağ M, Birkan B, Bumin G. Effects of swimming training on physical fitness and water orientation in autism. Pediatr Int. 2004;46(5):624-626; doi: 10.1111/j.1442-200x.2004.01938.x.

18. Thomas BR, Lafasakis M, Spector VJ. Brief report: using behavioral skills training to teach skateboarding skills to a child with autism spectrum disorder. Autism Dev Disord. 2016;46(12):3824-3829; doi: 10.1007/ s10803-016-2900-8.

19. Lochbaum M, Crews D. Viability of cardiorespiratory and muscular strength programs for the adolescent with autism. Complement Health Pract Rev. 2003;8(3):225-233; doi: $10.1177 / 1076167503252917$.

20. Todd T, Reid G. Increasing physical activity in individuals with autism. Focus Autism Other Dev Disabil. 2006;21(3): 167-176; doi: 10.1177/10883576060210030501.

21. Rogers L, Hemmeter ML, Wolery M. Using a constant time delay procedure to teach foundational swimming skills to children with autism. Top Early Child Spec Educ. 2010; 30(2):102-111; doi: 10.1177/0271121410369708.

22. Yanardag M, Akmanoglu N, Yilmaz I. The effectiveness of video prompting on teaching aquatic play skills for children with autism. Disabil Rehabil. 2013;35(1):47-56; doi: 10.3109/09638288.2012.687030.

23. Neely L, Rispoli M, Gerow S, Ninci J. Effects of antecedent exercise on academic engagement and stereotypy during instruction. Behav Modif. 2014;39(1):98-116; doi: 10.1177/0145445514552891.

24. De Milander M, Bradley S, Fourie R. Equine-assisted therapy as intervention for motor proficiency in children with autism spectrum disorder: case studies. S Afr J Res Sport Phys Educ Recreat. 2016;38(3):37-49.

25. Yu CCW, Wong SWL, Lo FSF, So RCH, Chan DFY. Study protocol: a randomized controlled trial study on the effect of a game-based exercise training program on promoting physical fitness and mental health in children with autism spectrum disorder. BMC Psychiatry. 2018;18(1):56; doi: 10.1186/s12888-018-1635-9.

26. Gabriels RL, Pan Z, Dechant B, Agnew JA, Brim N, Mesibov $\mathrm{G}$. Randomized controlled trial of therapeutic horseback riding in children and adolescents with autism spectrum disorder. J Am Acad Child Adolesc Psychiatry. 2015;54(7):541-549; doi: 10.1016/j.jaac.2015.04.007.

27. Toscano CVA, Carvalho HM, Ferreira JP. Exercise effects for children with autism spectrum disorder: metabolic health, autistic traits, and quality of life. Percept Mot Skills. 2018;125(1):126-146; doi:10.1177/0031512517743823.
28. Chan AS, Sze SL, Siu NY, Lau EM, Cheung MC. A chinese mind-body exercise improves self-control of children with autism: a randomized controlled trial. PLoS One. 2013;8(7):e68184; doi: 10.1371/journal.pone.0068184.

29. Bass MM, Duchowny CA, Llabre MM. The effect of therapeutic horseback riding on social functioning in children with autism. J Autism Dev Disord. 2009;39(9):12611271; doi: 10.1007/s10803-009-0734-3.

30. Kwon S, Sung IY, Ko EJ, Kim HS. Effects of therapeutic horseback riding on cognition and language in children with autism spectrum disorder or intellectual disability: a preliminary study. Ann Rehabil Med. 2019;43(3):279 288; doi: 10.5535/arm.2019.43.3.279.

31. Bahrami F, Movahedi A, Marandi SM, Sorensen C. The effect of karate techniques training on communication deficit of children with autism spectrum disorders. J Autism Dev Disord. 2016:46(3):978-986; doi: 10.1007/ s10803-015-2643-y.

32. Bahrami F, Movahedi A, Marandi SM, Abedi A. Kata techniques training consistently decreases stereotypy in children with autism spectrum disorder. Res Dev Disabil. 2012;33(4):1183-1193; doi: 10.1016/j.ridd.2012.01.018.

33. Yanardağ M, Birkan B, Yılmaz İ, Konukman F, Ağbuğa B, Lieberman $\mathrm{L}$. The effects of least-to-most prompting procedure in teaching basic tennis skills to children with autism. Kinesiology. 2011;43(1):44-55.

34. Prupas A, Reid G. Effects of exercise frequency on stereotypic behaviors of children with developmental disabilities. Educ Train Ment Ret Dev Disabil. 2001;36(2): 196-206.

35. García-Villamisar DA, Dattilo J. Effects of a leisure programme on quality of life and stress of individuals with ASD. J Intellect Disabil Res. 2010;54(7):611-619; doi: 10.1111/j.1365-2788.2010.01289.x.

36. Pitetti KH, Rendoff AD, Grover T, Beets MW. The efficacy of a 9-month treadmill walking program on the exercise capacity and weight reduction for adolescents with severe autism. J Autism Dev Disord. 2007;37(6):997-1006; doi: 10.1007/s10803-006-0238-3.

37. Anderson-Hanley C, Tureck K, Schneiderman RL. Autism and exergaming: effects on repetitive behaviors and cognition. Psychol Res Behav Manag. 2011;4:129-137; doi: 10.2147/PRBM.S24016.

38. Ennis E. The effects of a physical therapy-directed aquatic program on children with autism spectrum disorders. J Aquatic Phys Ther. 2011;19(1):4-10.

39. Rosenblatt LE, Gorantla S, Torres JA, Yarmush RS, Rao S, Park ER, et al. Relaxation response-based yoga improves functioning in young children with autism: a pilot study. J Altern Complement Med. 2011;17(11):10291035; doi: 10.1089/acm.2010.0834.

40. Morrison H, Roscoe EM, Atwell A. An evaluation of antecedent exercise on behavior maintained by automatic reinforcement using a three-component multiple schedule. J Appl Behav Anal. 2011;44(3):523-541; doi: 10.1901/ jaba.2011.44-523.

41. Ringenbach S, Lichtsinn K, Holzapfel S. Assisted Cycling Therapy (ACT) improves inhibition in adolescents with autism spectrum disorder. J Intellect Dev Disabil. 2015; 40(4):376-387; doi: 10.3109/13668250.2015.1080352.

42. Pan CY. The efficacy of an aquatic program on physical fitness and aquatic skills in children with and without autism spectrum disorders. Res Autism Spectrum Disord. 2011;5(1):657-665; doi: 10.1016/j.rasd.2010.08.001.

43. Fragala-Pinkham MA, Haley SM, O’Neil ME. Group swimming and aquatic exercise programme for children with 
autism spectrum disorders: a pilot study. Dev Neurorehabil.2011;14(4):230-241;doi:10.3109/17518423.2011. 575438.

44. Pan CY. Effects of water exercise swimming program on aquatic skills and social behaviors in children with autism spectrum disorders. Autism. 2010;14(1):9-28; doi: 10.1177/1362361309339496.

45. Movahedi A, Bahrami F, Marandi M, Abedi A. Improvement in social dysfunction of children with autism spectrum disorder following long term Kata techniques training. Res Autism Spectrum Disord.2013;7(9):1054-1061; doi: 10.1016/j.rasd.2013.04.012.

46. Kim Y, Todd T, Fujii T, Lim JC, Vrongistinos K, Jung T. Effects of Taekwondo intervention on balance in children with autism spectrum disorder. J Exerc Rehabil. 2016; 12(4):314-319; doi: 10.12965/jer.1632634.317.

47. Zhao M, Chen S. The effects of structured physical activity program on social interaction and communication for children with autism. Biomed Res Int. 2018;2018: 1825046; doi: 10.1155/2018/1825046.

48. Arzoglou D, Tsimaras V, Kotsikas G, Fotiadou E, Sidiropoulou $M$, Proios $M$, et al. The effect of a traditional dance training program on neuromuscular coordination of individuals with autism. J Phys Educ Sport. 2013;13(4): 563-569; doi: 10.7752/jpes.2013.04088.

49. Zamani Jam A, Talab RH, Sheikh M, Torabi F, Rafie F. The effect of 16 weeks gymnastic training on social skills and neuropsychiatric functions of autistic children. Sport Sci Health. 2018;14:209-214; doi: 10.1007/s11332017-0417-y.

50. Pan CY, Chu CH, Tsai CL, Sung MC, Huang CY, Ma WY. The impacts of physical activity intervention on physical and cognitive outcomes in children with autism spectrum disorder. Autism. 2017;21(2):190-202; doi: 10.1177/ 1362361316633562.

51. Wuang YP, Wang CC, Huang MH, Su CY. The effectiveness of simulated developmental horse-riding program in children with autism. Adapt Phys Activ Q. 2010;27(2): 113-126; doi: 10.1123/apaq.27.2.113.

52. Hayakawa K, Kobayashi K. Physical and motor skill training for children with intellectual disabilities. Percept MotSkills.2011;112(2):573-580; doi:10.2466/06.13.15. PMS.112.2.573-580.

53. Gabriels RL, Pan Z, Guérin NA, Dechant B, Mesibov G. Long-term effect of therapeutic horseback riding in youth with autism spectrum disorder: a randomized trial. Front Vet Sci. 2018;5:156; doi: 10.3389/fvets.2018.00156.

54. Kowaluk A, Woźniewski M. Physical activity and quality of life in children treated for leukaemia. Physiother Quart. 2018;26(2):9-18; doi: 10.5114/pq.2018.75994.

55. Czajka K, Sławińska T, Kołodziej M, Kochan K. Assessment of physical activity by pedometer in Polish preschool children. Hum Mov. 2015;16(1):15-19; doi: 10.1515/ humo-2015-0021.

56. Chagas DV, Batista LA. Comparison of health outcomes among children with different levels of motor competence. Hum Mov. 2017;18(2):56-61; doi: 10.1515/humo2017-0018.

57. Wójtowicz D, Roshko J, Ptak A, Dębiec-Bąk A, Skrzek A. The efficiency of rehabilitation for self-service eating in institutionalized children aged 2-6 years with mental and motor retardation. Physiother Quart. 2017;25(2):10-16; doi: 10.5114/pq.2018.73367.

58. Bergier J, Niźnikowska E, Bergier B, Acs P, Salonna F, Junger J. Differences in physical activity, nutritional be- girls from selected European countries. Hum Mov. 2017; 18(1):19-28; doi: 10.1515/humo-2017-0009.

59. Piłatowicz K, Zdunek MK, Molik B, Nowak AM, Marszałek J. Physical activity of children and youth with disabilities. Adv Rehab. 2018;32(4):45-54; doi: 10.5114/areh. 2018.83394.

60. Lewicka M, Kurylak A. Assessment of quality of life in patients with cerebral palsy. Pediatr Pol. 2019;94(2):119127; doi: 10.5114/polp.2019.85041.

61. Green D, Charman T, Pickles A, Chandler S, Loucas T, Simonoff E, et al. Impairment in movement skills of children with autistic spectrum disorders. Dev Med Child Neurol. 2009;51(4):311-316; doi: 10.1111/j.1469-8749. 2008.03242.x.

62. Manjiviona J, Prior M. Comparison of Asperger syndrome and high-functioning autistic children on a test of motor impairment. J Autism Dev Disord. 1995;25(1):2339; doi: 10.1007/BF02178165.

63. Downey R, Rapport MJK. Motor activity in children with autism: a review of current literature. Pediatr Phys Ther. 2012;24(1):2-20; doi: 10.1097/PEP.0b013e31823db95f.

64. Weimer AK, Schatz AM, Lincoln A, Ballantyne AO, Trauner DA. "Motor" impairment in Asperger syndrome: evidence for a deficit in proprioception. J Dev Behav Pediatr. 2001;22(2):92-101; doi: 10.1097/00004703-200104000 -00002 .

65. Fuentes CT, Mostofsky SH, Bastian AJ. Children with autism show specific handwriting impairments. Neurology. 2009;73(19):1532-1537; doi:10.1212/WNL.0b013e318 $1 \mathrm{c0d} 48 \mathrm{c}$.

66. Pan CY, Tsai CL, Chu CH. Fundamental movement skills in children diagnosed with autism spectrum disorders and attention deficit hyperactivity disorder. J Autism Dev Disord. 2009;39(12):1694-1705; doi: 10.1007/s10803009-0813-5.

67. Das Virgens Chagas D, Batista LA. Interrelationships among motor coordination, body fat percentage, and physical activity in adolescent girls. Hum Mov. 2015; 16(1):4-8; doi: 10.1515/humo-2015-0019.

68. Harmon J, Brusseau TA, Collier D, Lenz E. Habitual physical activity patterns of inner-city children. Hum Mov. 2013;14(4):305-309; doi: 10.2478/humo-2013-0036.

69. Lewicka M, Kurylak A. The quality of life of patients with cerebral palsy versus the general population. Pediatr Pol. 2019;94(5):288-292; doi: 10.5114/polp.2019.89864.

70. Srinivasan SM, Pescatello LS, Bhat AN. Current perspectives on physical activity and exercise recommendations for children and adolescents with autism spectrum disorders. Phys Ther. 2014;94(6):875-889; doi: 10.2522/ptj. 20130157. 